PROCEEDINGS OF THE

AMERICAN MATHEMATICAL SOCIETY

Volume 136, Number 9, September 2008, Pages 3051-3059

S 0002-9939(08)09277-0

Article electronically published on April 29, 2008

\title{
HECKE OPERATORS \\ FOR WEAKLY HOLOMORPHIC MODULAR FORMS AND SUPERSINGULAR CONGRUENCES
}

\author{
P. GUERZHOY
}

(Communicated by Ken Ono)

\begin{abstract}
We consider the action of Hecke operators on weakly holomorphic modular forms and a Hecke-equivariant duality between the spaces of holomorphic and weakly holomorphic cusp forms. As an application, we obtain congruences modulo supersingular primes, which connect Hecke eigenvalues and certain singular moduli.
\end{abstract}

\section{INTRODUCTION}

Let $M_{k}$ and $S_{k}$ be the $\mathbb{C}$-linear spaces of holomorphic modular and cusp forms correspondingly of weight $k$ with respect to the full modular group $S L_{2}(\mathbb{Z})$. Denote by $\widetilde{M_{k}}$ the $\mathbb{C}$-linear space of modular forms of weight $k$ which are holomorphic in the interior of the upper half-plane, and may have a pole at the cusp. We refer to the elements of $\widetilde{M_{k}}$ as weakly holomorphic modular forms. Any such form $f \in \widetilde{M_{k}}$ has a $q$-expansion

$$
f=\sum_{n \geq-h} a_{n} q^{n}, \quad q=\exp (2 \pi i z) \text { with } \Im(z)>0 .
$$

We say that $f$ is a cusp form if its $q$-expansion has no constant term, $a(0)=0$, and denote the $\mathbb{C}$-linear space of cusp forms of weight $k$ by $\widetilde{S_{k}}$.

For a (holomorphic) modular form $f$ of even positive integral weight $k$, one can consider Eichler integrals of two types:

$$
\mathcal{E}_{f}(z)=\int_{z}^{i \infty} f(\tau)(\tau-z)^{k-2} d \tau \text { and } \mathcal{F}_{f}(z)=\int_{-\bar{z}}^{i \infty} \overline{f(-\bar{\tau})}(\tau+z)^{k-2} d \tau .
$$

The transformation laws of both functions $\mathcal{E}_{f}(z)$ and $\mathcal{F}_{f}(z)$ define Eichler cohomology classes, and the corresponding maps from the space $S_{k}$ to the parabolic Eichler-Shimura cohomology are Hecke-equivariant (see, e.g., [6, Chapter 6]). In a recent paper [3, Theorem 1.1(2)], Bringmann and Ono prove, in particular, that there exists a function $\mathcal{G}$ which is holomorphic on the complex upper half-plane and transforms like $\mathcal{F}_{f}(z)$ under the action of $S L_{2}(\mathbb{Z})$ of weight $2-k$.

Note that a much more general result, which holds true also for congruence subgroups and half-integral weights, is actually proved in loc. cit. The authors remark

Received by the editors April 23, 2007, and, in revised form, July 16, 2007.

2000 Mathematics Subject Classification. Primary 11F37, 11F33.

This work is supported by NSF grant DMS-0700933.

(C)2008 American Mathematical Society Reverts to public domain 28 years from publication 
that this result is typical in the classical framework of Eichler cohomology. Recently, a close connection between the holomorphic function $\mathcal{G}$ and a Ramanujan mock theta-function was discovered in [4. The case $k=3 / 2$ and a certain congruence subgroup is considered in loc. cit., and striking applications are obtained. These achievements stimulate interest in other cases. In this paper we consider the case of even integral weight $k$ and full modular group $S L_{2}(\mathbb{Z})$.

Bol's identity [2] implies that the $(k-1)$-th derivative of the holomorphic function $\mathcal{G}$ must be a weakly holomorphic cusp form of weight $k$. The classical Eichler integral $\mathcal{E}_{f}(z)$ has a similar property: $\mathcal{E}_{f}^{(k-1)}=f$. This allows us to define (an analog of $) \mathcal{E}_{f}(z)$ for a weakly holomorphic cusp form $f$ as a $(k-1)$-th antiderivative of $f$ (the definition with an integral makes no sense since the integral diverges), and to obtain a Hecke-equivariant duality between the space $S_{k}$ and (a factor-space of) $\widetilde{S_{k}}$. Roughly, $\widetilde{f} \in \widetilde{S_{k}}$ is dual to $f \in S_{k}$ if $\mathcal{E}_{\tilde{f}}-\mathcal{F}_{f}$ transforms under the action of $S L_{2}(\mathbb{Z})$ like a modular form of weight $2-k$.

In this paper we consider a duality of this type from an elementary point of view, and obtain congruences which connect Hecke eigenvalues with certain singular moduli as an application. We formulate and discuss the results in Section 2, and provide the proofs in Section 3 of the paper.

\section{Statement And Discussion of RESults}

The Hecke operators $T_{p}$ with prime indices $p$ acting on $\widetilde{S_{k}}$ are defined in the usual way (cf. [8, Chapter II]). The same argument as in [8, Chapter II, Theorem 1.4] shows that if $f \in \widetilde{S_{k}}$ has a $q$-expansion (1), then $T_{p} f=\sum b_{n} q^{n}$ with

$$
b_{n}= \begin{cases}a_{p n} & \text { if } p \not n, \\ a_{p n}+p^{k-1} a_{n / p} & \text { if } p \mid n .\end{cases}
$$

Note that $\widetilde{S_{k}}$ may be non-empty for negative $k$, and is of infinite dimension if it is non-empty.

Denote by $D$ the differential operator

$$
D=q \frac{d}{d q}=\frac{1}{2 \pi i} \frac{d}{d z} .
$$

Assume that $k>2$, and is even. Although in general $D$ destroys modularity, Bol's identity implies that $D^{k-1}\left(\widetilde{M}_{-k+2}\right)$ is a linear subspace of $\widetilde{S_{k}}$. It is clear that $S_{k}$ is also a linear subspace of $\widetilde{S_{k}}$, and the two subspaces $D^{k-1}\left(\widetilde{M}_{-k+2}\right)$ and $S_{k}$ have a zero intersection since there are no holomorphic modular forms of negative weight $2-k$.

Denote by $\widehat{S_{k}}$ the quotient

$$
\widehat{S_{k}}=\widetilde{S_{k}} /\left(D^{k-1}\left(\widetilde{M}_{-k+2}\right) \oplus S_{k}\right) .
$$

A straightforward calculation which uses (2) proves the following commutation relation between Hecke operators and $D^{k-1}$ :

$$
T_{p} D^{k-1} g=p^{k-1} D^{k-1} T_{p} g,
$$

for every $g \in \widetilde{M_{-k+2}}$. This commutation relation along with the fact that Hecke operators act on $S_{k}$ implies that if $f \in \widetilde{S_{k}}$ is a representative of $\widehat{f} \in \widehat{S_{k}}$, then, for any prime $p$, the class in $\widehat{S_{k}}$ represented by $T_{p} f \in \widetilde{S_{k}}$ depends only on the class $\widehat{f}$, 
and does not depend on the choice of the representative $f$. This observation allows us to define the action of Hecke operators on the quotient space $\widehat{S_{k}}$.

Let $f \in \widetilde{S_{k}}$ with a $q$-expansion (1) be a representative of $\widehat{f} \in \widehat{S_{k}}$ and let $g=$ $\sum_{n>0} b_{n} q^{n} \in S_{k}$. Let

$$
\{\widehat{f}, g\}=\sum_{n=1}^{h} \frac{a_{-n} b_{n}}{n^{k-1}} .
$$

The product $\{\widehat{f}, g\}$ does not depend on the choice of the representative $f$. This is a part of the following result.

Theorem 1. The $\mathbb{C}$-linear space $\widehat{S_{k}}$ is finite-dimensional. More specifically,

$$
\operatorname{dim} \widehat{S_{k}}=\operatorname{dim} S_{k}
$$

The paring $\widehat{S_{k}} \times S_{k} \rightarrow \mathbb{C}$ defined by (41) is non-degenerate and Hecke equivariant. Specifically, for any prime $p$

$$
\left\{T_{p} \widehat{f}, g\right\}=\left\{\widehat{f}, T_{p} g\right\}
$$

The space $\widehat{S_{k}}$ admits a basis which consists of common eigenforms of Hecke operators; the eigenvalues are the same as for $S_{k}$.

As an application of this result, we derive some congruences which connect eigenvalues of Hecke operators acting on the space of cusp forms $S_{k}$ and certain singular moduli. In order to state the results, we introduce notation pertaining to the latter. We refer to [10, 5] for a detailed discussion. Let

$$
j=q^{-1}+744+1968844 q+\ldots
$$

denote the usual elliptic modular function (absolute invariant). For every positive integer $m$ let $j_{m}$ be the unique modular function which is holomorphic on the upper half-plane and has a $q$-expansion of the form $j_{m}(\tau)=q^{-m}+\mathcal{O}(q)$. In other words, $j_{m}=T_{p}(j-744)$. Note that

$$
j_{m}(\tau)=\phi_{m}(j(\tau))
$$

with a polynomial $\phi_{m} \in \mathbb{Z}[X]$ (Faber polynomial).

For a positive even integer $k \geq 12$, let $t=\operatorname{dim} S_{k}$, and let $f_{n}=\sum_{m>0} \lambda_{n, m} q^{m}$ with $n=1, \ldots, t$ be the basis of the space $S_{k}$ which consists of Hecke eigenforms $f_{n}$ normalized by the condition $\lambda_{n, 1}=1$. Then $T_{m} f_{n}=\lambda_{n, m} f_{n}$, and the $t \times t$ matrix

$$
\Lambda=\left(\lambda_{n, m}\right)_{n, m=1, \ldots, t}
$$

is invertible. Recall that the field $K=\mathbb{Q}\left(\lambda_{n, m}, n=1, \ldots, t ; m=1, \ldots\right)$ is an algebraic number field, and denote by $R$ its ring of integers.

Let $l>3$ be a prime. Let $\left(\mu_{m, n}^{\prime}\right)$ with $m, n=1, \ldots, t$ be the $t \times t$ matrix inverse to $\Lambda$. We renormalized this matrix, $\mu_{*, n}=M_{n} \mu_{*, n}^{\prime}$, multiplying its raws by appropriate factors such that all $\mu_{m, n}$ belong to $R$, and for any $n$ there exists $m$ such that $\mu_{m, n}$ is not divisible by $l$ in $R$. For algebraic integers, $\mu_{m, n}$ in particular, we denote by the same letters their images in $R / l R$. Moreover, for a rational integer $\gamma$ such that $\operatorname{ord}_{l}(\gamma) \geq 0$, the same letter $\gamma$ stands for the image of $\gamma$ in $\mathbb{Z} / l \mathbb{Z} \subset R / l R$. 
We also need the Bernoulli numbers $B_{r}$ and the arithmetic function $\sigma_{r-1}(m)=$ $\sum_{d \mid m} d^{r-1}$, which are involved into the $q$-expansion coefficients of normalized Eisenstein series $E_{r}$ of even weight $r$. More specifically, we put

$$
E_{r}= \begin{cases}1-\frac{2 r}{B_{r}} \sum_{n \geq 1} \sigma_{r-1}(n) q^{n} & \text { if } r \geq 4, \\ 1 & \text { if } r=0 .\end{cases}
$$

Recall that $E_{r} \in M_{r}$ for either $r=0$, or $r \geq 4$, even. We do not need any kind of $E_{2}$ here, and $M_{0}=\mathbb{C}$.

Theorem 2. Let $p>t=\operatorname{dim} S_{k}$ be a prime, and let $n \leq t$ be a positive integer.

Assume that the prime $l>\max (3, t)$ satisfies

$$
k \equiv 2 \bmod (l-1) .
$$

Let $s_{l} \in(\mathbb{Z} / l \mathbb{Z})[X] \subset(R / l R)[X]$ be the supersingular polynomial at $l$. The congruence

$$
\begin{aligned}
\sum_{m=1}^{t} \frac{\mu_{m, n}}{m^{k-1}} & \left(\phi_{p m}(X)-\lambda_{n, p} \phi_{m}(X)\right) \\
& \equiv \frac{2 k}{B_{k}}\left(p^{k-1}+1-\lambda_{n, p}\right) \sum_{m=1}^{t} \frac{\mu_{m, n} \sigma_{k-1}(m)}{m^{k-1}} \bmod s_{l}
\end{aligned}
$$

holds in $(R / l R)[X]$.

Remark 1 . The condition $p>t$ is technical; similar but less transparent congruences may be obtained when $p \leq t$.

Remark 2. We have to check that the left-hand side of (8) belongs to $R / l R$. Since $l>t$, this is clear if $B_{k} /(2 k)$ is invertible modulo $l$. The latter fact follows from the Kummer congruences. Indeed, condition (7) implies

$$
\left(1-l^{k-1}\right) \frac{B_{k}}{2 k} \equiv(1-l) \frac{B_{2}}{4}=\frac{1-l}{24} \not \equiv 0 \quad \bmod l .
$$

As an illustration of this result, we consider the simplest case when $k \in\{12,16$, $18,20,22,26\}$ which implies $t=\operatorname{dim} S_{k}=1$. Denote by

$$
g_{k}=f_{1}=\sum_{n \geq 1} \tau_{k}(n) q^{n}
$$

the unique cusp form $g_{k} \in S_{k}$ normalized by the condition $\tau_{k}(1)=1$. In particular, $\tau_{12}(n)$ is the Ramanujan $\tau$-function, and

$$
g_{12}=\Delta=q \prod_{n \geq 1}\left(1-q^{n}\right)^{24} .
$$

Since $\tau_{k}(n) \in \mathbb{Z}$ for every $n$, we have $K=\mathbb{Q}$, and $R=\mathbb{Z}$. The congruences (8) reduce to

$$
\phi_{p}(X)-\tau_{k}(p) \phi_{1}(X) \equiv \frac{2 k}{B_{k}}\left(\sigma_{k-1}(p)-\tau_{k}(p)\right) \quad \bmod s_{l} .
$$

Condition (7) for the weights $k$ under consideration implies $l \leq 19$. The supersingular polynomials $s_{l}$ for these primes are easily available (see, cf. [9, p. 37]). Let

$$
i=\sqrt{-1}, \quad \omega=\frac{1+i \sqrt{3}}{2}, \quad \xi=\frac{1+i \sqrt{7}}{2} .
$$


Then

$$
j(i)=1728, \quad j(\omega)=0, \quad j(\xi)=-3375 .
$$

We now plug in the values of $\tau=i, \omega$ or $\xi$ so that $s_{l} \equiv 0 \bmod l$, and summarize the congruences implied by (9) explicitly for every $3<l \leq 19$ in the following corollary.

Corollary 1. If $k=18,22$, or 26 , then

$$
j_{p}(\omega)-3 \tau_{k}(p) \equiv 4 \sigma_{k-1}(p) \quad \bmod 5 .
$$

If $k=20$ or 26 , then

$$
j_{p}(i)+6 \tau_{k}(p) \equiv 3 \sigma_{k-1}(p) \quad \bmod 7 .
$$

If $k=12$ or 22 , then

which imply

$$
\begin{gathered}
j_{p}(\omega)-2 \tau_{k}(p) \equiv 2 \sigma_{k-1}(p) \quad \bmod 11 \\
j_{p}(i)-3 \tau_{k}(p) \equiv 2 \sigma_{k-1}(p) \quad \bmod 11
\end{gathered}
$$

$$
\tau_{k}(p) \equiv j_{p}(i)-j_{p}(\omega) \quad \bmod 11
$$

If $k=26$, then

$$
j_{p}(\xi)+9 \tau_{k}(p) \equiv 11 \sigma_{k-1}(p) \quad \bmod 13
$$

If $k=18$, then

$$
\begin{aligned}
& j_{p}(\xi)-5 \tau_{k}(p) \equiv 7 \sigma_{k-1}(p) \quad \bmod 17 \\
& j_{p}(\omega)+3 \tau_{k}(p) \equiv 7 \sigma_{k-1}(p) \quad \bmod 17
\end{aligned}
$$

which imply

$$
\text { If } k=20 \text {, then }
$$

$$
8 \tau_{k}(p) \equiv j_{p}(\xi)-j_{p}(\omega) \bmod 17
$$

$$
\begin{gathered}
j_{p}(\xi)+\tau_{k}(p) \equiv 5 \sigma_{k-1}(p) \quad \bmod 19 \\
j_{p}(i)-10 \tau_{k}(p) \equiv 5 \sigma_{k-1}(p) \quad \bmod 19
\end{gathered}
$$

which imply

$$
11 \tau_{k}(p) \equiv j_{p}(i)-j_{p}(\xi) \quad \bmod 19
$$

Remark 3. Ken Ono pointed out to the author that an alternative way to obtain these congruences is to make use of [1, Corollary 4]. For example, the congruence (10) remains true for any $n \geq 1$, namely

$$
\tau_{12}(n) \equiv j_{n}(i)-j_{n}(\omega) \bmod 11
$$

not just for $n=p$, prime. This follows from identities [5, (1.9) and (1.10)], which are specializations of the above cited result, combined with $\Delta=\left(E_{4}^{3}-E_{6}^{2}\right) / 1728$ and the fact that $E_{4} E_{6}=E_{10} \equiv 1 \bmod 11$.

Remark 4. Our choice of points $i, \omega$ and $\xi$ was pretty much arbitrary. For example, $\xi=(1+i \sqrt{11}) / 2$ leaves all the above congruences unaltered.

In fact, not only congruences, but also some identities are available. We avoid general considerations, but provide a result in the special case $k=12$. We return back to the classical notations $\tau(n)=\tau_{12}(n)$, and note that the right-hand side of (9) reduces to the quantity

$$
a=\frac{65520}{691}\left(\tau(p)-\sigma_{11}(p)\right)
$$


Theorem 3. The following relations hold:

$$
\begin{gathered}
j_{p}(i)-264 \sum_{n=1}^{p-2} \sigma_{9}(n) j_{p-n}(i)-\left(264 \sigma_{9}(p-1)+\tau(p)\right) j_{1}(i)+264 \tau(p)=a, \\
j_{p}(\omega)-264 \sum_{n=1}^{p-2} \sigma_{9}(n) j_{p-n}(\omega)-\left(264 \sigma_{9}(p-1)+\tau(p)\right) j_{1}(\omega)+264 \tau(p)=a .
\end{gathered}
$$

One may obtain exact formulas for $\tau(p)$ and modulo 691 Ramanujan congruences as corollaries. As another corollary, we obtain congruences

$$
j_{p}(i) \equiv j_{p}(\omega) \equiv 0 \quad \bmod 24
$$

reducing the identities modulo $24=$ g.c.d. $(264,65520)$.

\section{Proofs}

Proof of Theorem 1. Let us first show that the pairing is well defined, namely that $\{\widehat{f}, g\}$ does not depend on the choice of a representative $f$ of $\widehat{f}$. It is sufficient to show that $\{f, g\}=0$ if either $f \in S_{k}$ or $f \in D^{k-1} \widetilde{M_{-k+2}}$. It is clear that $\{f, g\}=0$ if $f \in S_{k}$ since $f=\mathcal{O}(q)$ in this case. Assume that $f \in D^{k-1} \widetilde{M_{-k+2}}$. Then $f=D^{k-1} f_{1}$ with $f_{1} \in \widetilde{M_{-k+2}}$, and $\{f, g\}$ is the constant term of the $q$-expansion of $f_{1} g \in \widetilde{M}_{2}$. However, every weakly holomorphic modular form of weight 2 is a derivative of a polynomial in $j$, and therefore its constant term is zero.

Recall that

$$
t=\operatorname{dim} S_{k}= \begin{cases}{\left[\frac{k}{12}\right]-1} & \text { if } k \equiv 2 \quad \bmod 12, \\ {\left[\frac{k}{12}\right]} & \text { otherwise. }\end{cases}
$$

Let $f \in \widetilde{S_{k}}$ have the $q$-expansion $f=u q^{-h}+\mathcal{O}\left(q^{-h+1}\right)$ for a positive integer $h$ and $u \neq 0$. We claim that the class represented by $f$ in $\widehat{S_{k}}$ contains an element with $h \leq t$. Indeed, assume that $f$ is chosen in such a way that $h$ is minimal. If $h>t$, then $E_{12 h-k+2} \in M_{12 h-k+2}$ is defined by (6), and $g=E_{12 h-k+2} / \Delta^{h}$ belongs to $\widetilde{M_{-k+2}}$. Since $g=q^{-h}+\mathcal{O}\left(q^{-h+1}\right)$, the function $f+\left(u / h^{k-1}\right) D^{k-1} g=\mathcal{O}\left(q^{-h+1}\right)$ is a representative of the same class in $\widehat{S_{k}}$ as $f$. This contradiction to our choice of $f$ proves the inequality $\operatorname{dim} \widehat{S_{k}} \leq t$, since any element of $\widetilde{S_{k}}$ which has no principal part in the $q$-expansion belongs to $S_{k}$. Note that for any $h>0$, there exists $\alpha \in \mathbb{C}$ such that $E_{12 h+k} / \Delta^{h}-\alpha E_{k}=q^{-h}+\mathcal{O}\left(q^{-h+1}\right) \in \widetilde{S_{k}}$. The standard diagonalization procedure shows that if $\operatorname{dim} \widehat{S_{k}}<t$, then there exists $g=\mathcal{O}\left(q^{-t}\right) \in \widetilde{M_{-k+2}}$. It follows that $g \Delta^{t} \in M_{12 t-k+2}$, which is impossible since the latter space is empty because $12 t-k+2<0$. This proves the equality $\operatorname{dim} \widehat{S_{k}}=\operatorname{dim} S_{k}$. The fact that the pairing is non-degenerate follows at once from the above dioganalization procedure and a similar result about $S_{k}$ (see, e.g., [8, Chapter X, Theorem 4.4]). The fact that the pairing is Hecke-equivariant follows from (2). The existence of a basis of $\widehat{S_{k}}$ which consists of Hecke eigenforms and the claim about their eigenvalues now follow in a standard way from linear algebra.

Proof of Theorems 2 and 3, Let

$$
f_{n}^{*}=\sum_{n \geq-h} \mu_{m, n} q^{n}
$$


be a representative in $\widetilde{S_{k}}$ of the element in $\widehat{S_{k}}$ which is dual to $f_{n}$ with respect to pairing (4). In particular,

$$
\sum_{m=1}^{t} \frac{\mu_{m, n} \lambda_{r, m}}{m^{k-1}}=0 \text { if } r \neq n .
$$

It follows from Theorem 1 that the principal parts of the weakly holomorphic modular forms $T_{p} f_{n}^{*}$ and $\lambda_{n, p} f_{n}^{*}$ coincide modulo the principal part of $D^{k-1} g$ with a weakly holomorphic modular form $g \in \widetilde{M_{-k+2}}$. Taking into account (2), we compute (easily since $p>t$ ) the principal part of $T_{p} f_{n}^{*}$, and conclude that there exists $g \in \widetilde{M_{-k+2}}$ with a $q$-expansion

$$
g=\sum_{m=1}^{t} \mu_{m, n} \frac{1}{m^{k-1}} q^{-p m}-\lambda_{n, p} \sum_{m=1}^{t} \mu_{m, n} \frac{1}{m^{k-1}} q^{-m}+a+\mathcal{O}(q)
$$

for some $a \in \mathbb{C}$. In the special case when $k=12$, we have $t=1$, and

$$
g=q^{-p}-\tau(p) q^{-1}+a+\mathcal{O}(q) .
$$

We now determine the constant terms $a$ of these $q$-expansions. The product $g E_{k}$ is a weakly holomorphic modular form of weight 2 , therefore it must be a derivative of a polynomial in $j$; thus its constant term is zero. It follows that

$$
a=\left(\lambda_{n, p}-p^{k-1}-1\right) \frac{2 k}{B_{k}} \sum_{m=1}^{t} \frac{\mu_{m, n} \sigma_{k-1}(m)}{m^{k-1}},
$$

which reduces to (11) if $k=12$.

The product $g E_{k-2}$ is a weakly holomorphic modular form of weight zero, therefore, it is a polynomial in $j$. Put $\Psi(j)=g E_{k-2}$. For such a polynomial, the principal part of its $q$-expansion allows us to write the polynomial as a linear combination in $\phi_{m}$. Namely,

$$
\sum a_{m} q^{-m}+\mathcal{O}(q)=\sum a_{m} \phi_{m}(j)
$$

by definition of polynomials $\phi_{m}$. We now can finish the proof of Theorem 3 Indeed, since

$$
E_{10}=1-264 \sum_{n \geq 1} \sigma_{9}(n) q^{n}
$$

we obtain using (12)

$$
g E_{10}=\phi_{p}-264 \sum_{n=1}^{p-2} \sigma_{9}(n) \phi_{p-n}-\left(264 \sigma_{9}(p-1)+\tau(p)\right) \phi_{1}+264 \tau(p)+a .
$$

Since $E_{10}(\omega)=E_{10}(i)=0$, and $g$ is holomorphic on the upper half-plane, $g E_{10}(\omega)=$ $g E_{10}(i)=0$, we obtain the identities claimed in Theorem 3 making use of (5). A more general argument which finishes the proof of Theorem 2 will be similar to this one, because the values $j(i)$ and $j(\omega)$ are supersingular $j$-invariants at $l=11$. 
If $k-2=12 M+4 \delta+6 \varepsilon$ with a positive integer $M, \delta \in\{0,1,2\}$, and $\varepsilon \in\{0,1\}$, then we have a factorization

$$
E_{k-2}=\Delta^{M} E_{4}^{\delta} E_{6}^{\varepsilon} \Phi(j)
$$

with a polynomial $\Phi \in \mathbb{Q}[X]$. Condition (7) implies that $\Phi$ has $l$-integral coefficients and that the divisibility

$$
X^{\delta}(X-1728)^{\varepsilon} \Phi(X) \equiv 0 \quad \bmod s_{l}
$$

holds in $\mathbb{Z} / l \mathbb{Z}[X]$.

Note that $g \Delta^{p t} \in M_{12 p t+2-k}$ has $l$-integral $q$-expansion coefficients. This follows from our normalization of the matrix $\left(\mu_{m, n}\right)$, the inequality $12 p t>\operatorname{dim} M_{12 p t+2-k}$, and [8, Chapter X, Theorem 4.4]. Let $12 p t+2-k=12 M_{1}+4 \delta_{1}+6 \varepsilon_{1}$ with a positive integer $M, \delta_{1} \in\{0,1,2\}$, and $\varepsilon_{1} \in\{0,1\}$. As above, we have a factorization

$$
g \Delta^{p t}=\Delta^{M_{1}} E_{4}^{\delta_{1}} E_{6}^{\varepsilon_{1}} \Phi_{1}(j),
$$

and the polynomial $\Phi_{1} \in K[X]$ has $l$-integral coefficients since the $q$-expansion coefficients of $g \Delta^{p t}$ are $l$-integral. We thus have

$$
\Psi(j)=g E_{k-2}=\Delta^{M_{1}+M-p t} E_{4}^{\delta+\delta_{1}} E_{6}^{\varepsilon+\varepsilon_{1}} \Phi(j) \Phi_{1}(j) .
$$

It is now straightforward (see [9, 2.6,2.8.3]) to derive from (14) that the modulo $l$ reduction of $\Psi(X)$ is divisible by $s_{p}$ in $(R / l R)[X]$. This implies (8) due to (13) combined with the congruence $E_{k-2} \equiv 1 \bmod l$ coefficient-wise as $q$-expansions. The latter congruence follows from (7).

\section{ACKNOWLEDGEMENT}

The author is very grateful to Ken Ono for attracting the author's attention to this subject and for encouraging discussions and remarks.

\section{REFERENCES}

[1] Asai, T.; Kaneko, M.; Ninomiya, H., Zeros of certain modular functions and an application, Comment. Math. Univ. St. Paul. 46 (1997), no. 1, 93-101. MR1448475 (98e:11052)

[2] G. Bol, Invarianten linearer differentialgleichungen, Abh. Math. Sem. Univ. Hamburg 16 (1949), 1-28. MR0033411 (11:437a)

[3] Bringmann, K.; Ono, K., Lifting elliptic cusp forms to Maass forms with an application to partitions, Proceedings of the National Academy of Sciences, USA, 104 (2007), no. 10, 3725-3731. MR 2301875

[4] Bringmann, K.; Ono, K., Dyson's ranks and Maass forms, Annals of Mathematics, to appear.

[5] Bruinier, Jan H.; Kohnen, Winfried; Ono, Ken, The arithmetic of the values of modular functions and the divisors of modular forms, Compos. Math. 140 (2004), no. 3, 552-566. MR2041768 (2005h:11083)

[6] Hida, Haruzo, Elementary theory of $L$-functions and Eisenstein series, London Mathematical Society Student Texts, 26, Cambridge University Press, Cambridge, 1993. MR1216135 $(94 \mathrm{j}: 11044)$

[7] Kohnen, W.; Zagier, D., Modular forms with rational periods, Modular forms (Durham, 1983), 197-249, Ellis Horwood Ser. Math. Appl.: Statist. Oper. Res., Horwood, Chichester, 1984. MR803368 (87h:11043)

[8] Lang, Serge, Introduction to modular forms. With appendices by D. Zagier and Walter Feit. Corrected reprint of the 1976 original. Grundlehren der Mathematischen Wissenschaften [Fundamental Principles of Mathematical Sciences], 222, Springer-Verlag, Berlin, 1995. MR1363488 (96g:11037) 
[9] Ono, Ken, The web of modularity: Arithmetic of the coefficients of modular forms and $q$-series. CBMS Regional Conference Series in Mathematics, 102. Published for the Conference Board of the Mathematical Sciences, Washington, DC, by the American Mathematical Society, Providence, RI, 2004. MR2020489 (2005c:11053)

[10] Zagier, D., Traces of singular moduli, motives, polylogarithms and Hodge theory, Part I (Irvine, CA, 1998), 211-244, Int. Press Lect. Ser., 3, I, Int. Press, Somerville, MA, 2002. MR1977587 (2004h:11037)

Department of Mathematics, University of Hawail at Manoa, 2565 McCarthy Mall, Honolulu, HaWAir 96822-2273

E-mail address: pavel@math.hawaii.edu 